\title{
EARLY BRONZE AGE CHRONOLOGY: RADIOCARBON DATES AND CHRONOLOGICAL MODELS FROM TEL YARMUTH (ISRAEL)
}

\author{
Johanna Regev ${ }^{1,2} \cdot$ Pierre de Miroschedji $^{3} \cdot$ Elisabetta Boaretto ${ }^{2}$
}

\begin{abstract}
Over the years, 40 radiocarbon samples (charcoal and seeds) have been measured from the site of Tel Yarmuth. These samples originate from 3 major archaeological periods: Final Early Bronze Age (henceforth EB) I, EB II, and EB IIIBC. The samples are further on divided into 8 separate archaeological phases. Bayesian modeling analyses were performed on the data. Separate models were run with seeds and charcoals to detect a possible old-wood effect. Outliers were detected, and finally models with gaps were run to account for the lack of samples from 2 archaeological layers. The results suggest that at Tel Yarmuth the end of the EB II occurred 2950-2880 BC, and that the EB III ended at the latest $2450 \mathrm{BC}$, perhaps before $2500 \mathrm{BC}$. Although these dates are somewhat earlier than traditionally assumed, they are in close accordance with the new analysis of other ${ }^{14} \mathrm{C}$ dates for the Early Bronze Age in the southern Levant (Regev et al., these proceedings).
\end{abstract}

\section{INTRODUCTION}

The absolute chronology of the Early Bronze Age of the southern Levant is based on comparisons between pottery types securely placed within the local pottery sequence and comparable pottery types found in Egypt in historically dated contexts (Hennessy 1967; Ben-Tor 1991; Sowada 2009). A relative chronology has thus emerged, accepted by most scholars (e.g. Ben-Tor 1992; Mazar 1990; de Miroschedji 1999, in press a). It suggests that the Final EB IB period dates to the very end of the 4th millennium BC in Egypt (van den Brink and Levy 2002), while the EB II period is more or less synchronous with the first 2 dynasties, dated $3000-2700 \mathrm{BC}$ in the consensual "high" chronology (Kitchen 1991; Shaw 2000), and the EB III period with the Old Kingdom (dynasties 3 to 6), dated about 2700-2200 (Kitchen 1991; Shaw 2000). Other chronologies exist, which lower by 1 century or more the beginning of the 1st Dynasty (e.g. Hornung et al. 2006; Jiménez Serrano 2007; Wenke 2009). A chronology based on radiocarbon dates has recently been proposed by Bronk Ramsey et al. (2010). For the first half of the 3rd millennium, the latter chronology modifies only marginally the consensual High Chronology. As for the end of the EB III, it is conventionally placed around 2350-2300 BC, i.e. during the 6th Dynasty in Egypt (Mazar 1990; de Miroschedji 1999, in press b).

The basis for this absolute chronology is thus strictly historical and depends on the validity of the reconstruction of the Egyptian king lists and reign lengths inferred from written and archaeological sources. Given the incertitude surrounding this historical chronology, the establishment of a calibrated ${ }^{14} \mathrm{C}$ timescale is the only way to build on an independent basis the absolute chronology of the southern Levant.

Among the major Early Bronze Age sites of the southern Levant, Tel Yarmuth ranks prominently because of its size ( $16 \mathrm{ha}$ ), the large exposures of the Early Bronze Age strata exhibiting monumental architectural remains, the length of its stratigraphic record, and its many ${ }^{14} \mathrm{C}$ samples recovered from well-defined stratigraphic contexts. Tel Yamuth is thus an excellent site to carry out a detailed ${ }^{14} \mathrm{C}$ study of the Early Bronze Age with the aim of laying the ground for an absolute chronology.

\footnotetext{
'The Martin (Szusz) Department of Land of Israel Studies and Archaeology, Bar Ilan University, 52900 Ramat Gan, Israel. Corresponding author. Email: johanna.regev@ymail.com.

${ }^{2}$ Radiocarbon Dating and Cosmogenic Isotopes Laboratory, Kimmel Center of Archaeological Science, Weizmann Institute of Science, 76100 Rehovot, Israel.

${ }^{3}$ UMR 7041 (HAROC) - Archéologies et Sciences de l'Antiquité, Maison de l'archéologie et de l'ethnologie, Boîte 17, 21, allée de l'Université, 92023 Nanterre cédex, France.
}

C 2012 by the Arizona Board of Regents on behalf of the University of Arizona Proceedings of the 6th International Radiocarbon and Archaeology Symposium, edited by E Boaretto and N R Rebollo Franco RADIOCARBON, Vol 54, Nr 3-4, 2012, p 505-524 


\section{RADIOCARBON SAMPLES: PROVENANCE}

\section{The Site and Its Stratigraphy}

Tel Yarmuth is located $25 \mathrm{~km}$ southwest of Jerusalem (Figure 1). Nineteen seasons of excavations have been conducted at the site, one under the direction of A Ben-Tor in 1970 (Ben-Tor 1975), and 18 under the direction of $\mathrm{P}$ de Miroschedji since 1980 (see de Miroschedji et al. 1988; de Miroschedji 1999, 2003, 2008). Altogether, 13 areas of excavations (A-N) have been opened in the Lower City (Figure 2), some subdivided into several subareas (e.g. Area Ja to Jc).

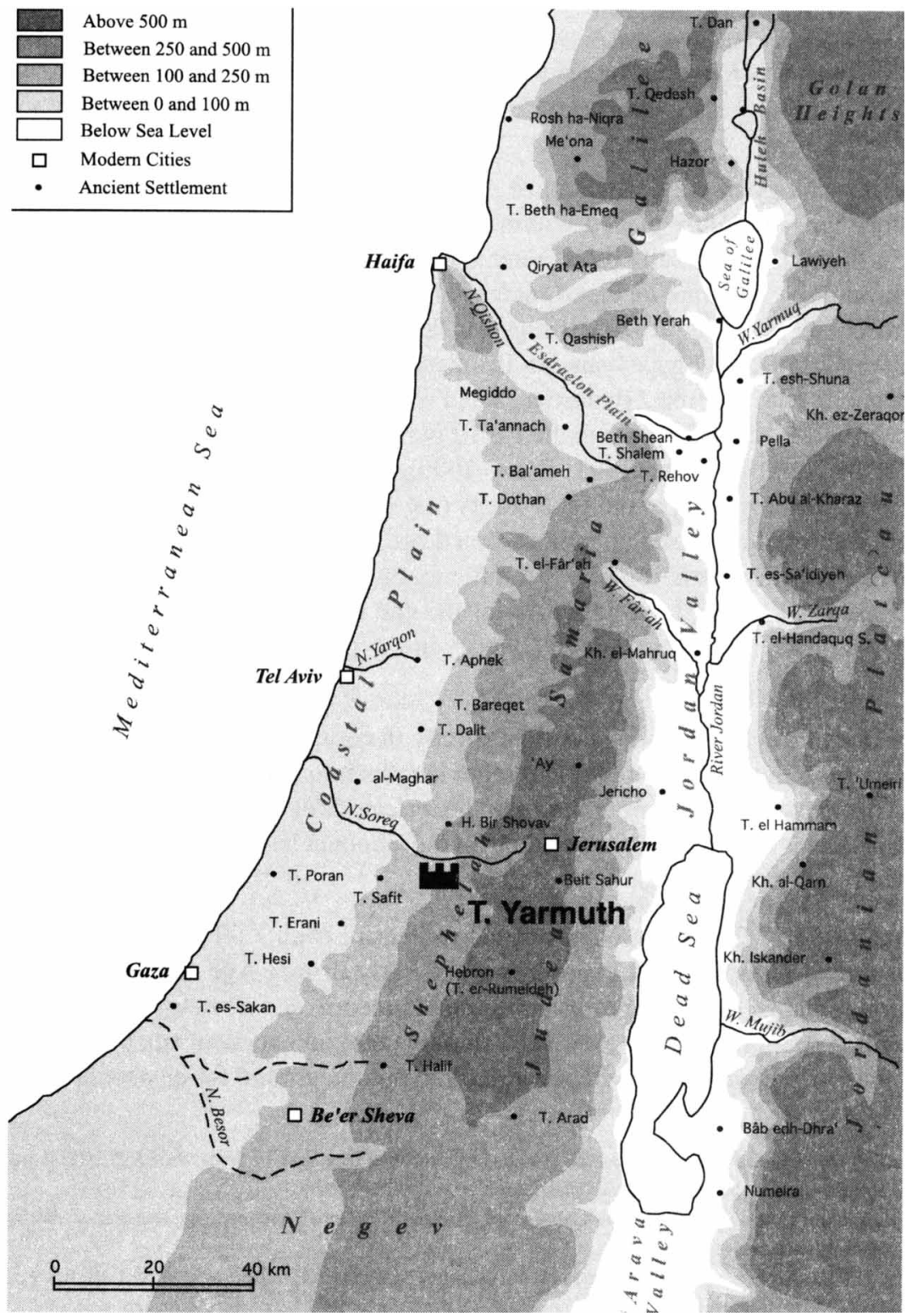

Figure 1 Map showing the location of Tel Yarmuth 


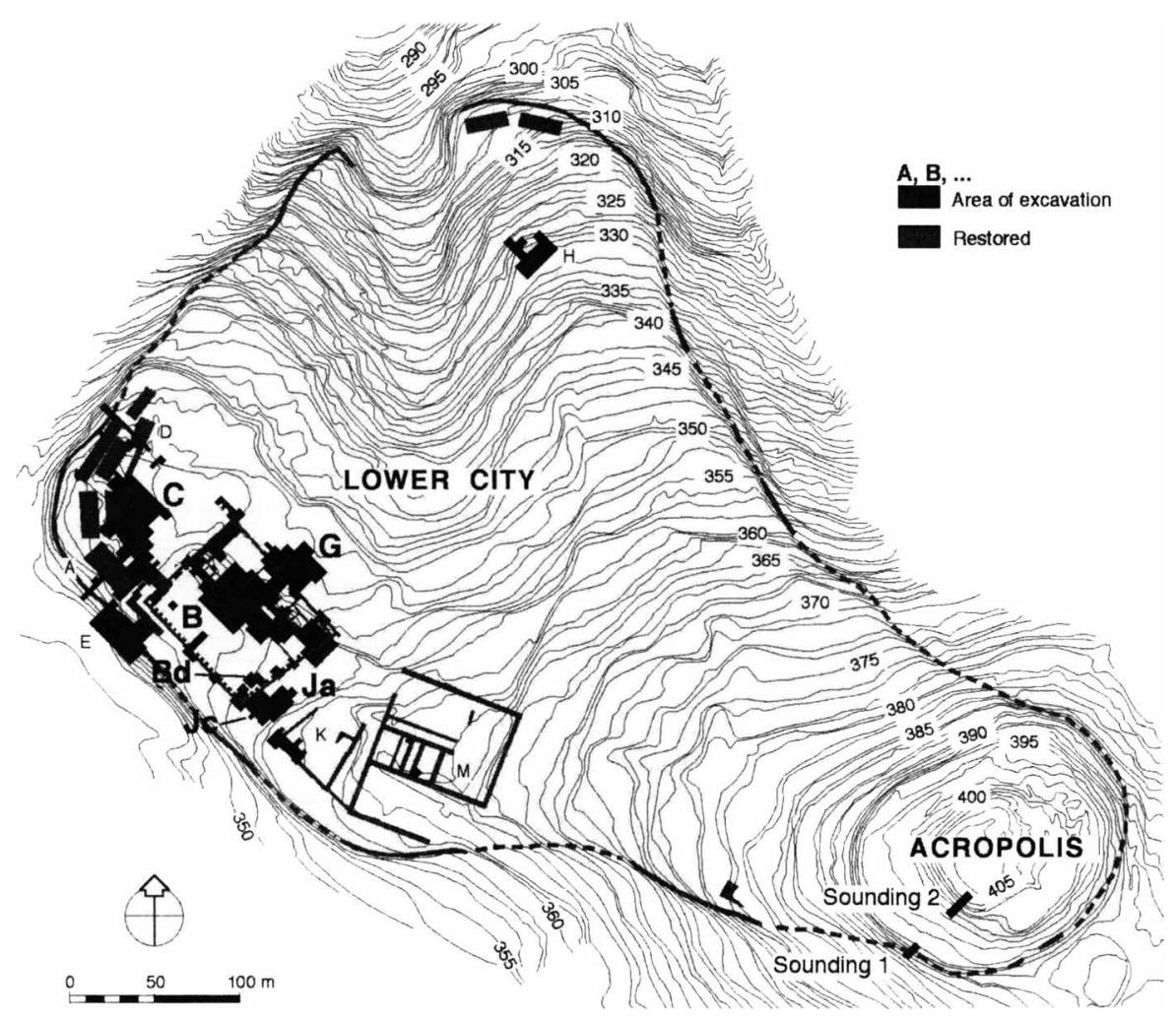

Figure 2 Plan showing the location of the excavation areas at Tel Yarmuth. The bold letters indicate the areas from which ${ }^{14} \mathrm{C}$ samples discussed in this paper originate.

These excavations have revealed a long sequence of occupation, stretching from the Early Bronze IB (the so-called "Erani C Phase," $3300 \mathrm{BC}$ in the conventional chronology) to the very end of the EB III (EB IIIC phase, 2350/2300 BC in the conventional chronology), represented by large-scale public and private architecture (including 2 successive palaces, a temple, 5 areas of domestic dwellings, and a possible oil factory), powerful fortifications ( 2 city walls, 8 bastions, 3 city gates), and a system of urban terracing.

The stratigraphic correlations between these excavation areas are based on similarities of archaeological material, mainly pottery, and/or on the relative stratigraphic position of the strata in relation to an architectural feature encompassing different areas, such as City Wall A or Palace B1.

\section{Contexts of the Samples}

The ${ }^{14} \mathrm{C}$ samples were collected during excavation, either directly by hand when they could be easily spotted or (in the case of seeds) after flotation of sediment samples. A few additional samples (e.g. RTT-5902 to -5905) were collected $a d$ hoc by E Boaretto and J Regev in 2006 after cleaning of stratigraphic sections and identification of clusters of seeds in controlled stratigraphic contexts. Although a large number of samples were collected, only 40 of them were selected for dating on the basis of the nature and quality of their archaeological context: only samples found on floors or in sealed contexts were used. The areas from which these samples originate are shown in bold letters in Figure 2 and their stratigraphic distribution is given in Table 1. Table 1 provides information on 
the stratigraphic and archaeological provenience of the 40 samples, and are arranged by area, in chronological order from the most ancient to the most recent archaeological contexts.

Table 1 Stratigraphic distribution of the ${ }^{14} \mathrm{C}$ samples of Tel Yarmuth. A vertical line between 2 numbers indicates that the samples were in direct superposition, a horizontal line that they come from the same layer. The numbers within a box correspond to samples retrieved from the same stratigraphic context, although not always in close proximity.

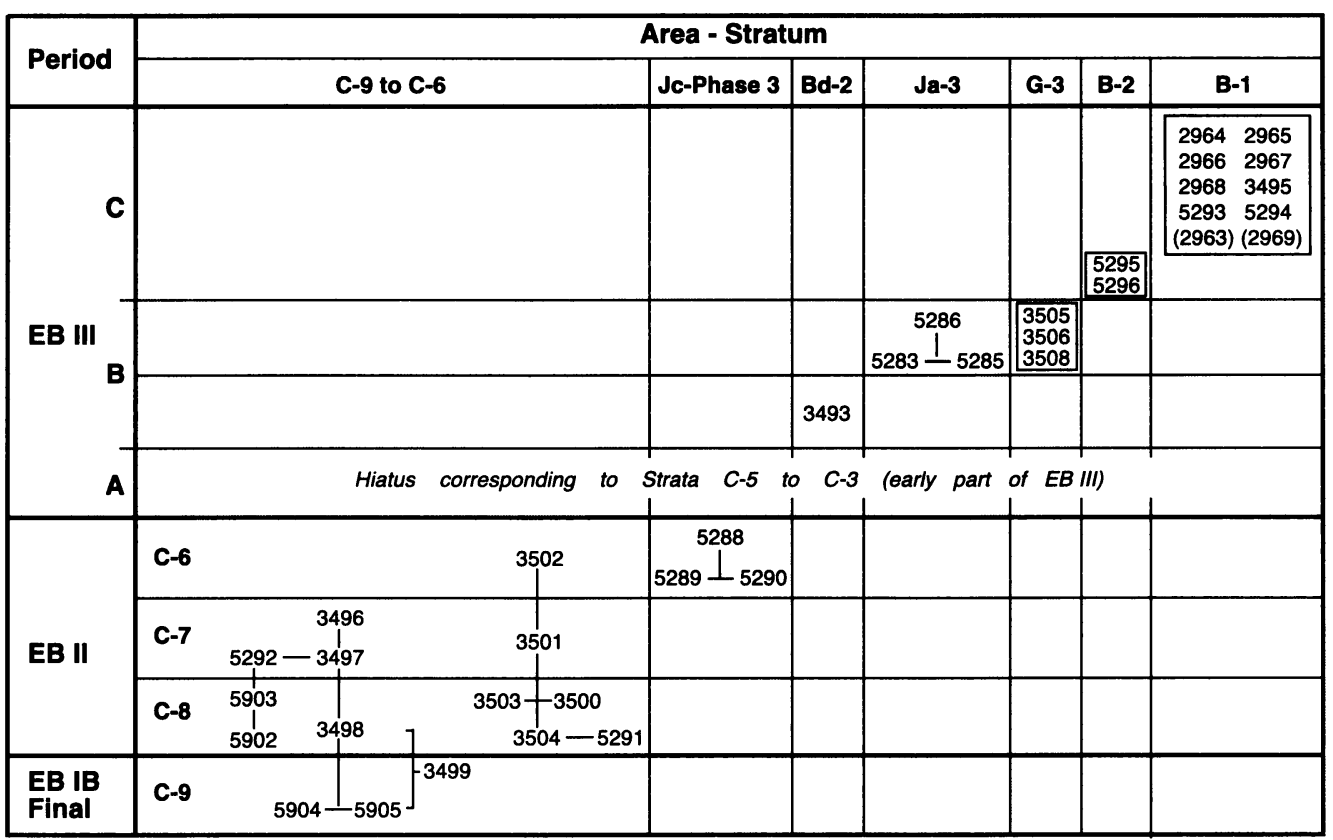

\section{Area C (15 Samples)}

Located in the west corner of the city, Area $C$ covered $950 \mathrm{~m}^{2}$ and revealed 9 strata (C-9 to C-1, from bottom to top). Stratum C-9, exposed in small probes only, consists of a thin layer $(\sim 0.20 \mathrm{~m})$ with occupation surfaces resting directly on bedrock and no associated architecture. The archaeological material is ascribed to the very end of the EB I period (Late EB IB). Sporadic finds of sherds dating to the EB IB [Erani C Phase] attest to an earlier occupation of the site but they were not found in clear stratigraphic context and have not yielded datable ${ }^{14} \mathrm{C}$ samples. This stratum yielded 2 samples of seeds (RTT-5904 and -5905). Stratum C-8 was also excavated in small soundings only and dates to the very beginning of the EB II. It belongs to an apparently unfortified settlement with some significant architectural remains. Seven samples originate from this stratum (RTA-3498, -3500, -3503, -3504; RTT-5291, -5902, -5903. The latter 3 samples were collected just beneath the foundation of City Wall A). One other sample (RTA-3499) comes from a context that may belong either to Stratum C-9 or C-8. The following strata C-7 and C-6 date to the traditional EB II period. Stratum C-7 is contemporaneous with the construction and early use of City Wall A. It has yielded 4 samples (RTA3496, -3497, -3501; RTT-5292). Only 1 sample (RTA-3502) comes from Stratum C-6. The uppermost strata, C-5 to C-1, all belong to the EB IIIA-B and have not yielded any samples appropriate for ${ }^{14} \mathrm{C}$ dating. 


\section{Subarea Jc (3 Samples)}

Subarea Jc corresponds to the part of Area J located outside (i.e. southwest) of City Wall A, which marks the limit of the first EB II settlement. A deep probe was excavated there through a fill intentionally deposited between City Wall A and City Wall B. This fill is ascribed to the second phase of the fortification system dated to a later part of the EB II (see de Miroschedji et al. 1988). The 3 samples (RTT-5288 to -5290) originate from layers of ashy sediments sealed by plastered surfaces in the upper part of the fill. The pottery assemblage suggests that they are contemporaneous with Stratum C-6 within the settlement.

\section{Subarea Bd (1 Sample)}

Subarea Bd is located along the southeast side of the main courtyard of Palace B1 and covers an area of $150 \mathrm{~m}^{2}$. Three strata were identified (Bd-3 to Bd-1), all dated to the EB III period. Stratum Bd-1 corresponds to Palace B1 (Stratum B-1), while Stratum Bd-2 (from which sample RTA 3493 was obtained) is contemporaneous with strata B-3 or B-4.

\section{Area G (4 Samples)}

Area $\mathrm{G}$ is situated to the southeast of Area $\mathrm{C}$ and extends along part of the northeastern side of Palace B1. It was excavated over $500 \mathrm{~m}^{2}$ and revealed 6 strata (G-6 to G-1) partly synchronous with those of Area B. Three samples (RTA-3505, -3506, -3508) originate from Stratum G-3, which is the extension of Stratum B-3 to the northeast of Area B. Stratum G-3 is represented by a series of domestic dwellings in which complete vessels were found in situ on floors. In its southwestern part, this stratum was violently destroyed, together with strata B-3, J-3, and $\mathrm{K}-2$, prior to the construction of Palace B2. A fourth sample (RTA-3507) from Stratum G-2 unexpectedly provided a "very old" date (see Table 1) and had to be discarded.

\section{Subarea Ja (5 Samples)}

Subarea Ja occupies a low terrace alongside the southeastern wall of Palace B1 and is limited to the southwest by the line of City Wall A. Partial excavations revealed 4 strata, J-1 to J-4 (from top to bottom). Strata J-1 and J-2 correspond, respectively, to strata B-1 and B-2. Stratum J-3 is coeval with Stratum B-3 and was also violently destroyed. Five samples (RTT-5283 to -5287) were retrieved from the destruction layer among vessels crushed on the floor under the fallen roof. Two of these samples (RTT-5284 and -5287), however, unexpectedly provided a post-bomb (i.e. modern) date and were clearly intrusive.

\section{Area B (12 Samples)}

The extent of Area B is defined by the limits of Palace B1. The stratigraphic sequence comprises 6 strata, B-1 to B-6, from top to bottom (de Miroschedji et al. 1988:31-3). Extensive excavations were carried out only for strata B-1 (= Palace B1), B-2 (= Palace B2), and B-3. The latter is equivalent to strata G-3 and J-3. Stratum B-2 corresponds to the first palace erected on the leveled ruins of a violently destroyed area. Since this palace was razed to the ground to be replaced by Palace B1, few floors were preserved. Two samples of seeds (RTT-5295, -5296) were nevertheless collected from a sound context (L.2632) sealed by a floor of the overlying Stratum B-1. The latter stratum yielded 8 samples (6 charcoals, 2 seeds) all retrieved from floors of Palace B-1, together with a large amount of complete vessels. Two additional samples from this stratum deserve a special note: RTT-2969 is a fragment of charcoal from a wooden post resting on a stone base in Locus 89 . This sample provided a date much earlier than that of the other samples, presumably because of the "old wood 
effect." The possibility of an "old wood effect" should also be considered in the case of sample RTT2963 since it consisted of charcoal from a wooden plank inserted into a threshold.

\section{Chronological Ordering of the Samples}

The ${ }^{14} \mathrm{C}$ samples can be arranged chronologically on the basis of the stratigraphic information described above (see Table 1). They document a continuous sequence from the Late EB IB to the end(?) of the EB II, and then from the second half or second third of the EB III until the end of the EB III. The current hypothesis is that the latest Early Bronze Age strata at Yarmuth (i.e. strata B-1, G-2, J-1, and K-1) immediately precede the late 3rd millennium abandonment of the site and date indeed from the very end of EB III period since no pottery later than the one found in these strata has been reported from any southern Levantine sites (for details see de Miroschedji 2000:319, 340). Hence, the sequence spanned by the ${ }^{14} \mathrm{C}$ dates incorporates a hiatus corresponding to strata $\mathrm{C}-5$ to C-3 in Area C, i.e. to the first third or first half of the EB III period, a phase labeled "EB IIIA." The length of this hiatus is impossible to estimate precisely; it could be 1 or 2 centuries.

\section{RADIOCARBON SAMPLES: PREPARATION AND ANALYSIS}

The 40 samples of charcoal and short-lived charred materials from Tel Yarmuth were dated at the Weizmann Institute Kimmel Center for Archaeological Sciences in Rehovot, Israel. The acid-baseacid protocol was used to remove contamination as described in Yizhaq et al. (2005). For the small samples like charred seeds, Fourier transform infrared (FTIR) analysis was performed after pretreatment in order to verify the absence of clay in the residual material (Yizhaq et al. 2005). After pretreatment, the ${ }^{14} \mathrm{C}$ concentrations were measured either by liquid scintillation counting (LSC) or by accelerator mass spectrometry (AMS) depending from the size of the sample after pretreatment. The data regarding the sample, context, type, and age are given in Table 2 in stratigraphic order.

Table 2 Results and details of the context of all the samples measured from Tel Yarmuth. The samples are listed in stratigraphic order. Short-lived material samples are marked with an asterisk after the sample number. Note the following abbreviations in the column "Archaeological context": Sq. = square; Str. = Stratum; L = Locus; B = Basket.

\begin{tabular}{|c|c|c|c|c|}
\hline Lab code & Age & $\begin{array}{l}\text { Calibrated } \pm 1 \sigma \\
\text { yr BC }\end{array}$ & $\begin{array}{l}\text { Calibrated } \pm 2 \sigma \\
\text { yr BC }\end{array}$ & Archaeological context \\
\hline RTT-5904* & $4455 \pm 60$ & $\begin{array}{l}3331(32.4 \%) 3215 \\
3186(7.4 \%) 3156 \\
3127(28.4 \%) 3023\end{array}$ & $\begin{array}{l}3346(86.4 \%) 3006 \\
2990(9.0 \%) 2930\end{array}$ & $\begin{array}{l}\text { Area C, Sq. Q13; Stratum C-9; L1038- } \\
\text { 2; field nr TY134; layer above bedrock } \\
\text { (same as RTT-5905); seeds }\end{array}$ \\
\hline RTT-5905* & $4345 \pm 60$ & $\begin{array}{l}3078(2.1 \%) 3073 \\
3024(66.1 \%) 2900\end{array}$ & 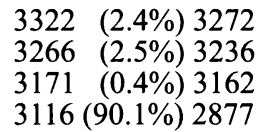 & $\begin{array}{l}\text { Area C, Sq. Q13; Stratum C-9; L1038- } \\
\text { 2; field nr TY141; layer above bedrock } \\
\text { (same as RTT-5904); seeds }\end{array}$ \\
\hline RTA-3499 & $4370 \pm 90$ & 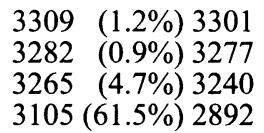 & $\begin{array}{l}3354(95.1 \%) 2872 \\
2799(0.2 \%) 2794 \\
2783 \quad(0.1 \%) 2781\end{array}$ & $\begin{array}{l}\text { Area C, Sq. P15; Stratum C-9 or C-8; } \\
\text { L392-1+2; B9293b; charcoal }\end{array}$ \\
\hline RTT-5902* & $4420 \pm 60$ & $\begin{array}{lll}3311 & (3.4 \%) & 3295 \\
3285 & (2.2 \%) & 3275 \\
3265 & (6.9 \%) & 3239 \\
3106 & (55.7 \%) & 2923\end{array}$ & 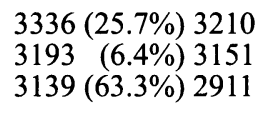 & $\begin{array}{l}\text { Area C, Sq. R12; Stratum C-8; base of } \\
\text { L1055; field nr TY 118; seeds }\end{array}$ \\
\hline RTT-5903* & $4330 \pm 55$ & $3014(68.2 \%) 2897$ & $\begin{array}{l}3264(1.0 \%) 3246 \\
3101(94.4 \%) 2874\end{array}$ & $\begin{array}{l}\text { Area C, Sq. R12; Stratum C-8; L1052 } \\
\text { (floor at the junction of L1052A and B); } \\
\text { field nr TY 119; seeds }\end{array}$ \\
\hline RTA-3498 & $4385 \pm 55$ & $\begin{array}{l}3090(16.1 \%) 3047 \\
3033(52.1 \%) 2917\end{array}$ & $\begin{array}{l}3327(11.3 \%) 3231 \\
3225(0.5 \%) 3219 \\
3176(1.4 \%) 3160 \\
3121(82.3 \%) 2896\end{array}$ & $\begin{array}{l}\text { Area C, Sq. Q13; Stratum C-8; L1038- } \\
\text { 1+floor a; B9281b (just above RTT- } \\
5904 \text { and -5905); charcoal }\end{array}$ \\
\hline
\end{tabular}


Table 2 Results and details of the context of all the samples measured from Tel Yarmuth. The samples are listed in stratigraphic order. Short-lived material samples are marked with an asterisk after the sample number. Note the following abbreviations in the column "Archaeological context": Sq. = square; Str. = Stratum; L = Locus; B $=$ Basket. (Continued)

\begin{tabular}{|c|c|c|c|c|}
\hline Lab code & Age & $\begin{array}{l}\text { Calibrated } \pm 1 \sigma \\
\text { yr BC }\end{array}$ & $\begin{array}{l}\text { Calibrated } \pm 2 \sigma \\
\text { yr BC }\end{array}$ & Archaeological context \\
\hline RTA-3504 & $4330 \pm 45$ & $\begin{array}{l}3011(22.2 \%) 2977 \\
2971(11.0 \%) 2949 \\
2944(35.0 \%) 2898\end{array}$ & $\begin{array}{l}3090(6.7 \%) 3051 \\
3031(88.7 \%) 2882\end{array}$ & $\begin{array}{l}\text { Area C, Sq. N18 Stratum C-8; L925; } \\
\text { B9403; charcoal }\end{array}$ \\
\hline RTT-5291* & $4300 \pm 40$ & $\begin{array}{l}3007(9.1 \%) 2989 \\
2931(59.1 \%) 2883\end{array}$ & $3024(95.4 \%) 2876$ & $\begin{array}{l}\text { Area C, Sq. O17; Stratum C-8; L922-1; } \\
\text { B9363; seeds, Scorpiurus sp. }\end{array}$ \\
\hline RTA-3503 & $4390 \pm 50$ & $\begin{array}{l}3089(15.8 \%) 3051 \\
3031(52.4 \%) 2920\end{array}$ & $\begin{array}{l}3325(10.4 \%) 3234 \\
3173(1.0 \%) 3161 \\
3118(83.9 \%) 2901\end{array}$ & $\begin{array}{l}\text { Area C, Sq. N18; Stratum C-8; L910-2; } \\
\text { B9389; charcoal }\end{array}$ \\
\hline RTA-3500 & $4360 \pm 60$ & $\begin{array}{l}3084(6.8 \%) 3066 \\
3028(61.4 \%) 2905\end{array}$ & 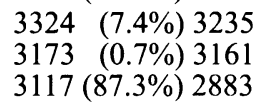 & $\begin{array}{l}\text { Area C, Sq. N17; Stratum C-8; L913; } \\
\text { B9214; charcoal }\end{array}$ \\
\hline RTT-5292* & $4380 \pm 40$ & $\begin{array}{l}3079(4.5 \%) 3071 \\
3025(63.7 \%) 2921\end{array}$ & $\begin{array}{l}3264(1.6 \%) 3247 \\
3101(93.8 \%) 2902\end{array}$ & $\begin{array}{l}\text { Area C, Sq. R13; Stratum C-7; L609- } \\
\text { surface e; B9222b; seeds, Lolizm sp. }\end{array}$ \\
\hline RTA-3497 & $4325 \pm 50$ & $\begin{array}{l}3011(21.1 \%) 2977 \\
2971(11.1 \%) 2949 \\
2944(36.1 \%) 2895\end{array}$ & $\begin{array}{l}3091(8.2 \%) 3043 \\
3038(87.2 \%) \\
2879\end{array}$ & $\begin{array}{l}\text { Area C, Sq. Q14; Stratum C-7; L1030- } \\
\text { floor c=L1051; B9279b; charcoal }\end{array}$ \\
\hline RTA-3496 & $4365 \pm 50$ & $\begin{array}{l}3079(3.7 \%) 3071 \\
3025(64.5 \%) 2911\end{array}$ & 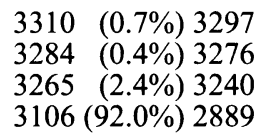 & $\begin{array}{l}\text { Area C, Sq. Q13-14; Stratum C-7; } \\
\text { L1030-floor a; B9227b; charcoal }\end{array}$ \\
\hline RTA-3501 & $4425 \pm 55$ & 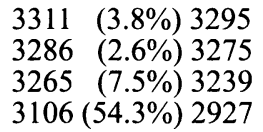 & 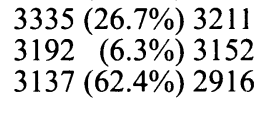 & $\begin{array}{l}\text { Area C, Sq. N17; Stratum C-7; L657- } \\
\text { 3+floor c; charcoal }\end{array}$ \\
\hline RTA-3502 & $4470 \pm 90$ & $\begin{array}{l}3339(32.3 \%) 3206 \\
3196(26.2 \%) 3082 \\
3069(9.7 \%) 3026\end{array}$ & $3370(95.4 \%) 2910$ & $\begin{array}{l}\text { Area C, Sq. O17; Stratum C-6A; L922- } \\
\text { 1+Tr.931-2; B9383; charcoal }\end{array}$ \\
\hline RTT-5289* & $4410 \pm 40$ & $\begin{array}{l}3095(43.1 \%) 3006 \\
2991(25.1 \%) 2930\end{array}$ & 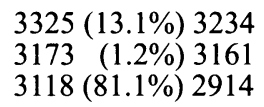 & $\begin{array}{l}\text { Area Jc, Sq. H42; Phase Jc-3; L2821; } \\
\text { B17567 (same as RTT-5290); seeds, } \\
\text { Lolium sp. }\end{array}$ \\
\hline RTT-5290* & $4360 \pm 40$ & $3016(68.2 \%) 2916$ & $3091(95.4 \%) 2900$ & $\begin{array}{l}\text { Area Jc, Sq. H42; Phase Jc-3; L2821; } \\
\text { B17567 (same as RTT-5289); seeds, ce- } \\
\text { reals }\end{array}$ \\
\hline RTT-5288* & $4340 \pm 40$ & $\begin{array}{l}3011(24.0 \%) 2978 \\
2971(13.8 \%) 2949 \\
2944(30.4 \%) 2905\end{array}$ & $\begin{array}{l}3087(5.6 \%) 3061 \\
3030(89.8 \%) 2890\end{array}$ & $\begin{array}{l}\text { Area Jc, Sq. H42; Phase Jc-3; L2819-1 } \\
\text { (layer of black ashy earth sealed by } \\
\text { plastered surface); B17551; seeds, Lens }\end{array}$ \\
\hline RTA-3493 & $4300 \pm 55$ & 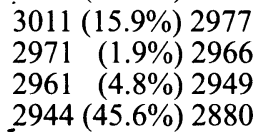 & 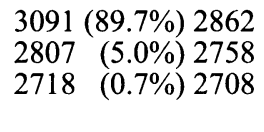 & $\begin{array}{l}\text { Area Bd, Sq. L39; Stratum Bd-2 (corre- } \\
\text { sponding to Str. J-4); L1805-1; } \\
\text { B14039; charcoal }\end{array}$ \\
\hline RTA-3505 & $4135 \pm 50$ & $\begin{array}{l}2870(19.7 \%) 2800 \\
2760(48.5 \%) 2620\end{array}$ & $2880(95.4 \%) 2570$ & $\begin{array}{l}\text { Area G, Sq. AB33; Stratum G-3; } \\
\text { L1298-floor a; B12276; charcoal }\end{array}$ \\
\hline RTA-3506 & $4100 \pm 50$ & $\begin{array}{l}2855(15.7 \%) 2812 \\
2747(6.9 \%) 2725 \\
2697(45.6 \%) 2577\end{array}$ & $\begin{array}{l}2873(90.6 \%) \\
2524 \quad(4.8 \%) \\
2496\end{array}$ & $\begin{array}{l}\text { Area G, Sq. X31; Stratum G-3; L1279- } \\
1 ; \text { B12321; charcoal }\end{array}$ \\
\hline RT-3508 & $4342 \pm 50$ & $3013(68.2 \%) 2905$ & $3096(95.4 \%) 2885$ & $\begin{array}{l}\text { Area G, Sq. X31; Stratum G-3; paved } \\
\text { threshold between L1234 and L1219; } \\
\text { B12510; charcoal }\end{array}$ \\
\hline RTT-5283* & $4150 \pm 70$ & $\begin{array}{l}2873(12.8 \%) 2834 \\
2818(51.2 \%) 2662 \\
2649 \quad(4.2 \%) 2635\end{array}$ & $\begin{array}{l}2896(93.9 \%) \\
2516 \quad(1.5 \%) 2569 \\
2500\end{array}$ & $\begin{array}{l}\text { Area Ja, Sq. K41-42; Stratum J-3; } \\
\text { L2 } 104 \text { (black ashes on floor } 2104-a) \\
\text { B17561; seeds }\end{array}$ \\
\hline RTT-5285* & $4240 \pm 70$ & 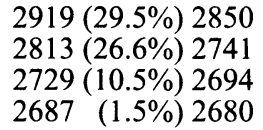 & $3021(95.4 \%) 2620$ & $\begin{array}{l}\text { Area Ja, Sq. K44; Stratum J-3; L2137- } \\
2 \text { + floor b; B17583; seeds }\end{array}$ \\
\hline
\end{tabular}


Table 2 Results and details of the context of all the samples measured from Tel Yarmuth. The samples are listed in stratigraphic order. Short-lived material samples are marked with an asterisk after the sample number. Note the following abbreviations in the column "Archaeological context": Sq. = square; Str. = Stratum; L = Locus; B = Basket. (Continued)

\begin{tabular}{|c|c|c|c|c|}
\hline Lab code & Age & $\begin{array}{l}\text { Calibrated } \pm 1 \sigma \\
\mathrm{yr} \mathrm{BC}\end{array}$ & $\begin{array}{l}\text { Calibrated } \pm 2 \sigma \\
\text { yr BC }\end{array}$ & Archaeological context \\
\hline RTT-5286* & $3795 \pm 40$ & $\begin{array}{l}2290(54.8 \%) 2195 \\
2172(13.4 \%) 2145\end{array}$ & 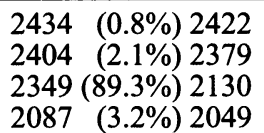 & $\begin{array}{l}\text { Area Ja, Sq. K41-42; Stratum J-3; } \\
\text { L2137-1+2 (ashy earth); B17611; } \\
\text { seeds, cereals }\end{array}$ \\
\hline RTT-5295* & $4135 \pm 40$ & $\begin{array}{l}2864(13.4 \%) 2830 \\
2822(5.8 \%) 2807 \\
2759(16.8 \%) 2717 \\
2711(32.2 \%) 2628\end{array}$ & $\begin{array}{l}2875(89.7 \%) 2617 \\
2611 \quad(5.7 \%) 2581\end{array}$ & $\begin{array}{l}\text { Area Ba, Sq. Q26; Stratum B-2; L2632; } \\
\text { B17159 (beneath RTT 5293 and RT } \\
5294 \text { of L89; same as RTT 5296); } \\
\text { seeds, cereals }\end{array}$ \\
\hline RTT-5296* & $4250 \pm 40$ & $\begin{array}{l}2911(55.1 \%) 2870 \\
2802(13.1 \%) 2778\end{array}$ & 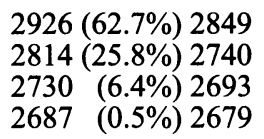 & $\begin{array}{l}\text { Area Ba, Sq. Q26; Stratum B-2; L2632; } \\
\text { B17151+B17159+B17169 (beneath } \\
\text { RTT } 5293 \text { of L89; same as RTT 5295); } \\
\text { seeds, cereals }\end{array}$ \\
\hline RT-2969 & $4420 \pm 25$ & $\begin{array}{l}3261(1.7 \%) 3257 \\
3097(65.4 \%) 3012 \\
2948 \quad(1.1 \%) 2945\end{array}$ & 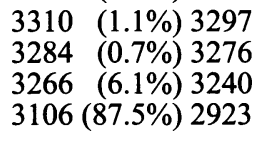 & $\begin{array}{l}\text { Area Ba, Sq. Q26; Stratum B-1; Palace } \\
\text { B1, L89-floor a (above RTT 5295 and } \\
5296 \text { from L2632); B13431. Fragment } \\
\text { of a wooden post: probable old wood } \\
\text { effect; charcoal }\end{array}$ \\
\hline RT-2966 & $4215 \pm 65$ & $\begin{array}{l}2903(22.7 \%) 2850 \\
2813(30.7 \%) 2742 \\
2729(12.9 \%) 2694 \\
2687(2.0 \%) 2680\end{array}$ & $\begin{array}{l}2924(93.2 \%) 2617 \\
2611 \quad(2.2 \%) 2581\end{array}$ & $\begin{array}{l}\text { Area Ba, Sq. Q28; Stratum B-1; Palace } \\
\text { B1, L1619; B13333; charcoal }\end{array}$ \\
\hline RTT-5293* & $4210 \pm 40$ & 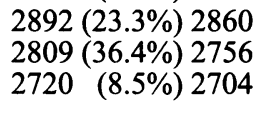 & $\begin{array}{l}2905(31.6 \%) 2835 \\
2816(63.8 \%) 2668\end{array}$ & $\begin{array}{l}\text { Area Ba, Sq. Q26; Stratum B-1; Palace } \\
\text { B1, L89- floor a (seeds on floor); } \\
\text { B17150 (same as RTT 5294); seeds, ce- } \\
\text { reals }\end{array}$ \\
\hline RTT-5294* & $4180 \pm 40$ & $\begin{array}{l}2880(14.4 \%) 2851 \\
2813(37.3 \%) 2743 \\
2727(15.6 \%) 2695 \\
2683(0.9 \%) 2681\end{array}$ & $\begin{array}{l}2891(22.1 \%) 2831 \\
2821(73.3 \%) 2631\end{array}$ & $\begin{array}{l}\text { Area Ba, Sq. Q26; Stratum B-1; Palace } \\
\text { B1, L89 (seeds on floor); B17168 } \\
\text { (same as RTT 5293); seeds, cereals }\end{array}$ \\
\hline RT-2963 & $4115 \pm 35$ & $\begin{array}{l}2860(20.3 \%) 2810 \\
2750(9.4 \%) 2720 \\
2700(38.5 \%) 2580\end{array}$ & $\begin{array}{l}2880(24.5 \%) 2800 \\
2780(70.9 \%) 2570\end{array}$ & $\begin{array}{l}\text { Area Bb, Sq. T37; Stratum B-1; Palace } \\
\text { B1, L2018 floor; B15053 or B15059? } \\
\text { Charcoal from plank on threshold: pos- } \\
\text { sible old wood effect; charcoal }\end{array}$ \\
\hline RT-2964 & $4105 \pm 50$ & $\begin{array}{l}2860(16.3 \%) 2810 \\
2750(7.4 \%) 2720 \\
2700(44.5 \%) 2570\end{array}$ & $\begin{array}{l}2880(91.4 \%) 2560 \\
2530(4.0 \%) 2490\end{array}$ & $\begin{array}{l}\text { Area Bb, Sq. S 38; Stratum B-1; Palace } \\
\text { B1, L2025 floor; B15112; charcoal }\end{array}$ \\
\hline RT-3495 & $4100 \pm 23$ & $\begin{array}{l}2840(14.4 \%) 2810 \\
2670(53.8 \%) 2570\end{array}$ & $\begin{array}{l}2860(22.0 \%) 2800 \\
2750(6.9 \%) 2720 \\
2700(66.5 \%) 2570\end{array}$ & $\begin{array}{l}\text { Area Bh, Sq. T40; Stratum B-1; Palace } \\
\text { B1, L1970; B15666. Sample taken } \\
\text { from layer of debris on the floor; char- } \\
\text { coal }\end{array}$ \\
\hline RT-2967 & $4035 \pm 95$ & 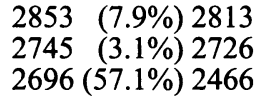 & $\begin{array}{l}2878(94.6 \%) 2339 \\
2322(0.8 \%) 2309\end{array}$ & $\begin{array}{l}\text { Area Bb, Sq. S36-37; Stratum B-1; Pal- } \\
\text { ace B1, L2012-1; B15174; charcoal }\end{array}$ \\
\hline RT-2968 & $3980 \pm 40$ & $\begin{array}{l}2569(40.4 \%) 2517 \\
2500(27.8 \%) 2467\end{array}$ & $\begin{array}{l}2618(0.7 \%) 2610 \\
2581(90.3 \%) 2400 \\
2382(4.4 \%) 2347\end{array}$ & $\begin{array}{l}\text { Area Ba, Sq. R 25; Palace B1, Stratum } \\
\text { B-1; L80-1 to } 3 \text { + floor a; B13071; char- } \\
\text { coal }\end{array}$ \\
\hline RT-2965 & $3565 \pm 40$ & $\begin{array}{l}2009(2.3 \%) 2002 \\
1977(62.5 \%) 1879 \\
1839(3.4 \%) 1830\end{array}$ & $\begin{array}{l}2026(76.5 \%) 1865 \\
1849(18.9 \%) 1773\end{array}$ & $\begin{array}{l}\text { Area Ba, Sq. R29; Stratum B-1; Palace } \\
\text { B1, L1647-1+floor a; B13341; Palace } \\
\text { B1; charcoal }\end{array}$ \\
\hline RTA-3507 & $45,150 \pm 550$ & & & $\begin{array}{l}\text { Area G, Stratum G-2; L1418-floor c; } \\
\text { B12490; charcoal }\end{array}$ \\
\hline RTT-5284* & $\begin{array}{l}131.3 \pm 05 \\
\mathrm{pMC}\end{array}$ & modern & & $\begin{array}{l}\text { Area Ja; Stratum J-3; L2104; B17575; } \\
\text { Thymelaeaceae }\end{array}$ \\
\hline RTT-5287* & $\begin{array}{l}110.0 \pm 03 \\
\mathrm{pMC}\end{array}$ & modern & & $\begin{array}{l}\text { Area Ja; Stratum J-3; L 2819; B17550; } \\
\text { Pisum }\end{array}$ \\
\hline
\end{tabular}


${ }^{14} \mathrm{C}$ ages are reported in conventional ${ }^{14} \mathrm{C}$ yr BP (AD 1950) in accordance with international convention (Stuiver and Polach 1977). Thus, all calculated ${ }^{14} \mathrm{C}$ ages have been corrected for fractionation as to refer the results to be equivalent with the standard $\delta^{13} \mathrm{C}$ value of $-25 \%$. All the samples have $\delta^{13} \mathrm{C}$ values between $-22 \%$ and $-27 \%$. The only exception is sample RTT-3507 $\left(\delta^{13} \mathrm{C}=-29.0 \%\right.$ ) All ${ }^{14} \mathrm{C}$ ages were calibrated using OxCal v 4.1.6 (Bronk Ramsey 2009a) and the IntCal09 data (Reimer et al. 2009) calibration curve. Analysis and modeling of the dates based on archaeological information was performed using the Bayesian method and the mathematical tools as given in OxCal v 4.1.6.

Two of the samples, RTT-5284 and RTT-5287, both originating from Subarea Ja, were found to be modern (intrusive), and 1 sample, RTA-3507, provided an age of 45,150 $\pm 550 \mathrm{yr}$ uncalibrated. This sample was probably a piece of asphalt used in antiquity (Aufderheide et al. 2004). After removal of these 3 samples, 37 dates were used for building a chronology for Tel Yarmuth: 3 samples $(2$ seeds, 1 charcoal) originate from "Late EB IB" contexts, 15 samples ( 7 seeds, 8 charcoals) from EB II contexts, 7 samples ( 3 seeds, 4 charcoals) from EB IIIB contexts, and 12 samples ( 4 seeds, 8 charcoals) from EB IIIC contexts (Table 2).

\section{MODELING}

Various Bayesian models were applied in order to determine the most reliable dates of transition between the archaeological layers. Four types of models were applied: Simple model, Detailed model, a model with an imposed gap, and a model with gap with terminus post quem (henceforth TPQ) of $2900 \pm 10 \mathrm{yr}$ BC for the beginning of the gap. Each of these models was again separated into models of seeds only and charcoal only in order to evaluate the possible old-wood effect. Outliers identified after the modeling were excluded and the model was run without the outliers. In case an end of a previous phase is given by a short-lived sample that is an outlier, while the beginning of the following phase is given by charcoal sample, we preferred to remove the charcoal because of the possibility of the old-wood effect.

As a general rule, any single date that had an agreement of less than $60 \%$ was considered an outlier. However, some samples close to the $60 \%$ cut-off will be just above or below the $60 \%$ cut-off when a model is run a few times. If more than 1 sample was identified as an outlier, the removal was done stepwise, namely the one with the lowest agreement was removed first. The model was run again after removal of each outlier, to verify if the removal of the sample(s) would cause a change in the agreement of the rest of the samples.

The models are built using phases or sequences, following the archaeological information. If a model was built using phases, the samples within each phase were ordered from oldest to youngest. This does not indicate that the samples were necessarily found in that order in the archaeological layers. If the model was built using sequences, the samples are ordered according to archaeological stratigraphic order. OxCal v 4.1.6 (Bronk Ramsey 2009a) software was used to calculate the transitions.

\section{Structure of the Models}

Simple models: The data were separated according to their association to Final EB I, EB II, or EB III cultural materials. In the Simple model, the dates were ordered as phases, since it was not possible to know the exact stratigraphic order of the samples within each period. Inside the phases, dates were ordered from old to young independent of the stratigraphy. The Final EB I to EB II transition is "contiguous," implying that as the previous period ended, the following started (see OxCal v 4.1). The EB II to EB IIIB transition is "sequential" due to the gap in the ${ }^{14} \mathrm{C}$ data. This model should pro- 
vide the transition between the Final EB I and EB II and the end range for EB II and beginning range for EB IIIB.

Detailed models: In this model, the data are separated according to the detailed stratigraphic sequence of the site. Several samples from layers C-8, C-7, C-6A, and Jc-3 had additional information about their inner ordering within the layer indicating which samples were found one above the other. Accordingly, for these layers the samples were ordered as sequences, while the samples that had no additional indication of their relative placement in the strata were placed in the sequence according to their ${ }^{14} \mathrm{C}$ age. Where no information on the relative placement was available, the samples were ordered in phases, also from oldest to youngest, even though this does not affect the modeling. The transitions between phases or sequences are contiguous, except in the case of the EB IIEB IIIB boundary, where it is sequential due to the absence of datable ${ }^{14} \mathrm{C}$ samples from strata $\mathrm{C}-5$, C-4, C-3, covering the EB IIIA period.

Models with a gap: The length of the EB IIIA period at Tel Yarmuth lacking ${ }^{14} \mathrm{C}$ dates was estimated to be between 100 to $200 \mathrm{yr}$. Therefore, separate models were run by inserting a gap of $150 \pm$ 50 yr between the EB II and EB IIIB layers. These models have the same structure as the Simple and the Detailed models described above.

Models with a gap and a terminus post quem (TPQ): A terminus post quem was inserted before the $150 \pm 50 \mathrm{yr}$ gap. This was done in order to determine whether it would be possible to stabilize the end of the EB II period around $2900 \mathrm{BC}$.

\section{RESULTS}

In Figures 3, 4, 6, and 7, all the models are shown with all the dates, including the outliers. Outliers are identified by the code $[\mathrm{P}]$ and a question mark after the sample number and they are plotted in dark gray only (e.g. samples RTT-5291 and RT-2969 in Figure 3). Even though they are seen in the plot, they do not affect the model. The general agreement of the model is given on top of the multiplot, written as [Amodel:xxx] (e.g. in the Simple model Figure 3 the general agreement is 123). After each ${ }^{14} \mathrm{C}$ date, the sample number appears first, and nearby is given the amount of agreement [A:xxx] of each individual date within the model (e.g. in Figure 3 sample RTT-5904 has an A value of 95). The light gray single plots describe the whole span of possible dates as determined by the calibration combined with laboratory error. The probability distributions marked with dark gray define the modeled results.

\section{EB I-II and EB II-III Transitions According to the Simple and Detailed Models}

The Simple model has 2 outliers (RTT-5291, RT-2969). The agreement of the model was 94 before their removal and 123 after (Table 3). As a result, the EB II end and EB IIIB begin dates were lowered by about $30 \mathrm{yr}$. According to the Simple model, the EB I-II transition took place between 30302970 BC and the EB II ended by 3000-2930 BC (Figure 3). The EB IIIB began between 2960-2910 $\mathrm{BC}$. These dates are significantly higher than the conventionally accepted dates (see above). If only seed samples are used, the EB I-II lower limit of the transition becomes younger by $20 \mathrm{yr}$. The EB II ends between 2980-2910 BC, and EB IIIB starts between 2950-2850 BC, up to 60 yr later than when charcoal samples are also used.

In the Detailed model, the major transitions turn out to be very similar to the Simple model, but they are more spread by few decades (Figure 4, Table 3). The EB I-II transition range is 3050-2980 BC, being up to $20 \mathrm{yr}$ older than in the Simple model. The EB II ends 2960-2910 BC, which is a date up to 40 yr younger than in the Simple model. The EB IIIB begins also $\sim 30$ yr later (2930-2880 BC). 
Table 3 Modeled ranges for EB I-II transition, EB II end, and EB IIIB begin ranges in all the models. The transition limit is given in relation to the $\pm 1 \sigma$.

\begin{tabular}{|c|c|c|c|c|c|c|}
\hline Name of the model & EB I-II & EB II end & $\begin{array}{l}\text { EB IIIB } \\
\text { begin }\end{array}$ & Dates & Outliers removed & $\begin{array}{l}\text { Agree- } \\
\text { ment }\end{array}$ \\
\hline Simple with outliers & $3030-2990$ & $3010-2960$ & $2990-2940$ & 37 & - & 94 \\
\hline Simple & $3030-2970$ & $3000-2930$ & $2960-2910$ & 35 & 5291,2969 & 123 \\
\hline Simple seeds & $3030-2950$ & $2980-2910$ & $2950-2850$ & 16 & 5291 & 90 \\
\hline Simple charcoal & $3040-2970$ & $3010-2940$ & $2970-2910$ & 21 & 2969 & 119 \\
\hline $\begin{array}{l}\text { Simple with gap } \\
150 \pm 50 \mathrm{yr}\end{array}$ & $3030-2980$ & $3010-2950$ & $2840-2770$ & 33 & $5291,2969,3508,3493,5296$ & 110 \\
\hline Simple seeds with gap & $3040-2970$ & $3010-2940$ & $2830-2750$ & 14 & 5291,5296 & 83 \\
\hline $\begin{array}{l}\text { Simple charcoal with } \\
\text { gap }\end{array}$ & $3040-2960$ & $3000-2920$ & $2820-2720$ & 18 & $2969,3508,3493$ & 112 \\
\hline $\begin{array}{l}\text { Simple with gap } \\
150 \pm 50 \mathrm{yr} \\
\text { TPQ } 2900 \pm 10 \mathrm{BC}\end{array}$ & $3010-2930$ & $2920-2890$ & $2770-2720$ & 33 & $2969,3508,3493,5285$ & 87 \\
\hline $\begin{array}{l}\text { Simple seeds with gap } \\
150 \pm 50 \mathrm{yr} \\
\text { TPQ } 2900 \pm 10 \mathrm{BC}\end{array}$ & $3020-2930$ & $2920-2880$ & $2760-2700$ & 14 & 5296,5293 & 74 \\
\hline $\begin{array}{l}\text { Simple charcoal with } \\
\text { gap } 150 \pm 50 \mathrm{yr} \\
\text { TPQ } 2900 \pm 10 \mathrm{BC}\end{array}$ & $3050-2940$ & $2920-2880$ & $2760-2700$ & 18 & $2969,3508,3493$ & 104 \\
\hline Detailed & $3050-2980$ & $2960-2910$ & $2930-2880$ & 32 & $5291,3508,5286,5296,2969$ & 122 \\
\hline Detailed seeds & $3040-2950$ & $2950-2890$ & $2920-2860$ & 14 & 5286,5296 & 125 \\
\hline Detailed charcoal & $3060-2980$ & $2970-2910$ & $2940-2880$ & 19 & 3508,2969 & 110 \\
\hline $\begin{array}{l}\text { Detailed with gap } \\
150 \pm 50 \mathrm{yr}\end{array}$ & $3050-2980$ & $2970-2910$ & $2810-2740$ & 30 & $\begin{array}{l}5291,3508,5286,5296, \\
2969,3493,5293\end{array}$ & 106 \\
\hline $\begin{array}{l}\text { Detailed with gap } \\
150 \pm 50 \mathrm{yr} \\
\text { TPQ } 2900 \pm 10 \mathrm{BC}\end{array}$ & $3050-2980$ & $2920-2880$ & $2760-2710$ & 28 & $\begin{array}{l}5291,3508,5286,5296, \\
2969,3493,5293,2966,5294\end{array}$ & 125 \\
\hline
\end{tabular}

Since the Detailed model comprises 8 separate phases, it is very efficient in pinpointing outliers. Out of the 37 samples, 5 are below $60 \%$ agreement, and were accordingly removed from the final model. The samples RTT-5286 and -2969 are clear outliers, and cannot be placed in the model in the given stratigraphic position. As pointed out by the excavator (see above), the sample RT-2969 has very probably a significant old-wood effect since it is a fragment of charcoal from a wooden post resting on a stone base. The seed sample RTT-5286 from Stratum Ja-3 is probably intrusive. It should be noted that out of the 5 samples dated from this area, 2 were found modern, indicating some clear disturbance in this vicinity. The seed sample RTT-529I found under City Wall A in Area C is somewhat surprisingly young. Sample RT-3508 from Stratum G-3 is likely affected by an old-wood effect, and sample RTT-5296 from Stratum B-2 is not a striking outlier, although it may be an intrusion from the overlying layer.

The "Final EB I"-II transition in all 6 Simple and Detailed models (Simple, Simple seeds only, Simple charcoal only, Detailed, Detailed seeds only, and Detailed charcoal only) is more or less the same: it starts between 3060-3030 BC and ends between 2980-2950 BC (see Figure 5, black bars, and Table 4). The 30 -yr variability in ranges is probably due to a minor old-wood effect since it corresponds to the differences between the "seeds only" and the "charcoal only" models. In the same 6 models, the "EB II end" ranges have slightly greater variance, from 3010-2950 to 2940-2890 BC (Figure 5, white bars). In this transition as well, the "charcoal only" results are older by 20-60 yr. Similar discrepancies between short-lived material and samples with a possible old-wood effect are apparent in the dates for the beginning of EB IIIB: 2970-2920 BC and 2910-2850 BC (Figure 5, gray bars). 


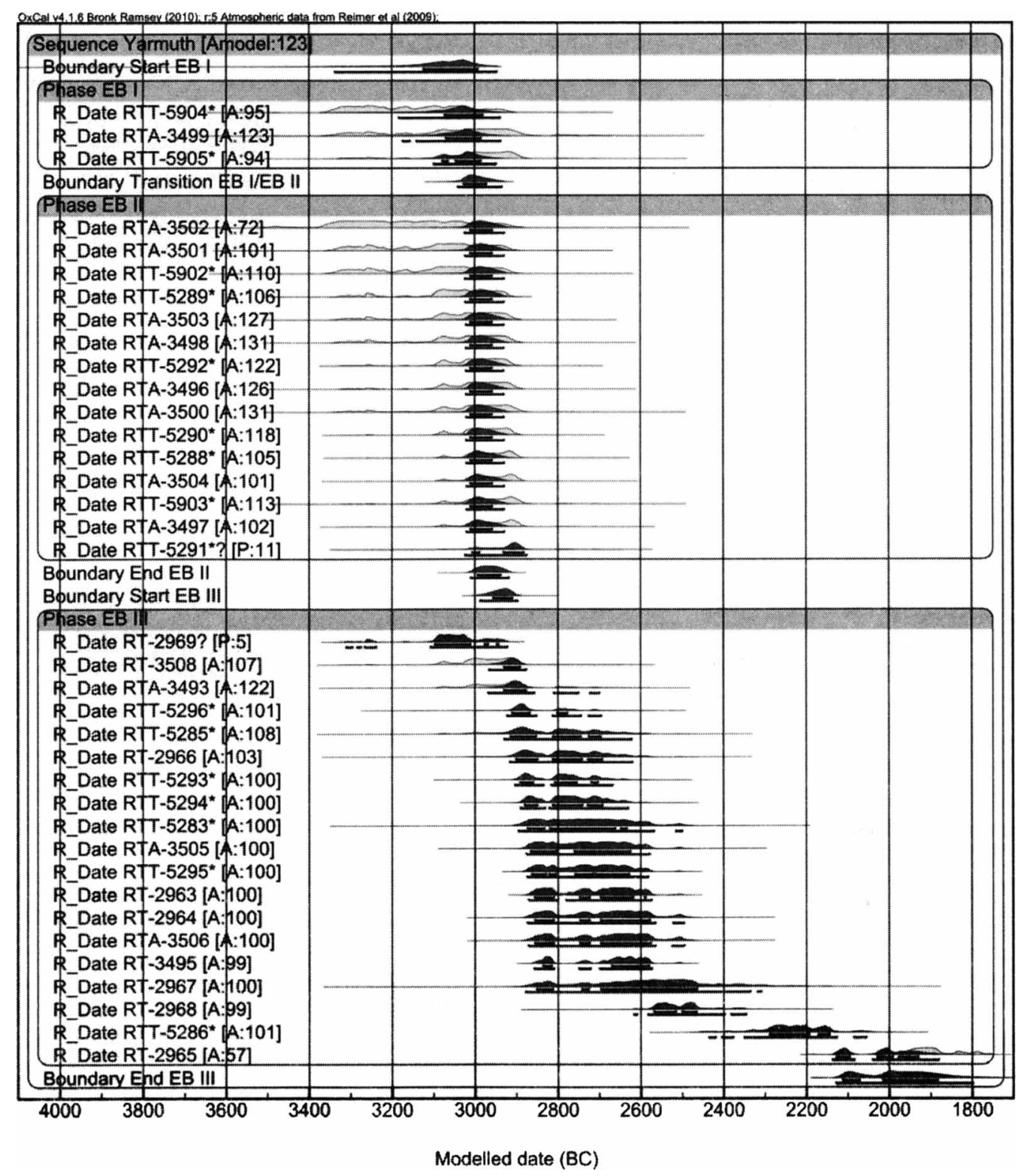

Figure 3 The Simple model: Simple "Final EB I," EB II, EB III transitions, using seeds and charcoal samples together. Two outliers are marked with a "P" and excluded from the analysis.

Even when the latest date of $2890 \mathrm{BC}$ for the end of EB II is taken as the actual end of the period, the dates remain older than currently accepted by $\sim 200$ yr. Furthermore, the beginning of EB IIIB starts before $2850 \mathrm{BC}$, leaving a short duration of $40 \mathrm{yr}$ for the EB IIIA period (instead of the 100$200 \mathrm{yr}$ accepted conventionally). If a longer EB IIIA period is more likely, the EB II period could have ended as early as $3010 \mathrm{BC}$ based on our results, leaving $160 \mathrm{yr}$ of duration for the EB IIIA period. It is important to stress that in any combination these dates are older than expected, and allow a short duration for the EB IIIA period. For this reason, it was decided to test the insertion of a gap of $150 \pm 50 \mathrm{yr}$ to the model between the end of EB II and the beginning of EB IIIB. 


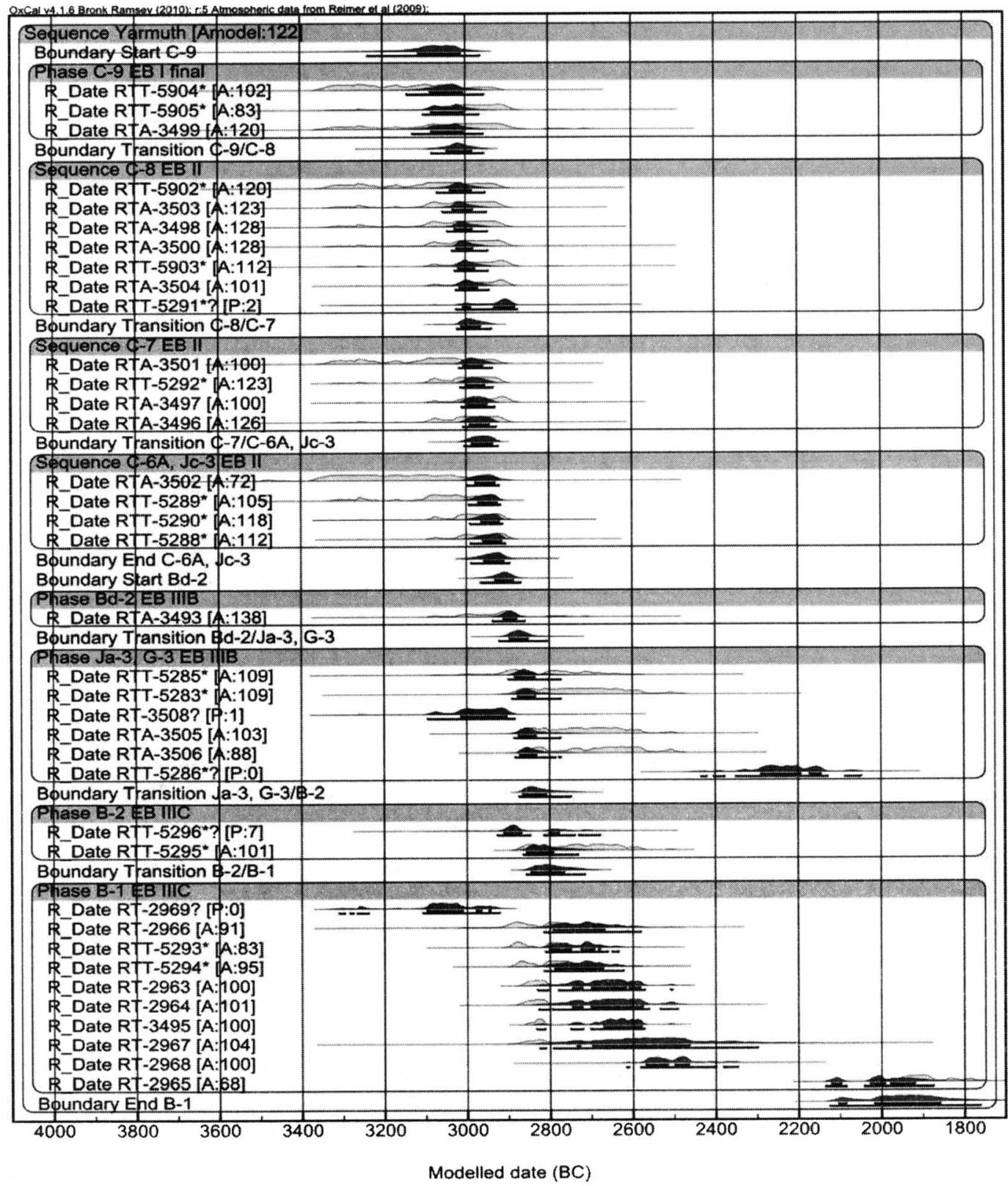

Figure 4 The "Detailed" model

\section{Models with Inserted Gap and TPQ}

The insertion of the $150 \pm 50 \mathrm{yr}$ gap after the end of the EB II period in the Simple model shifted the end of that period upward by only 10-20 yr (3010-2950 BC) compared to the original Simple model (3000-2930 BC). In contrast, the beginning of the EB IIIB period was shifted downward by as much as $120-140 \mathrm{yr}$, to $2840-2770 \mathrm{BC}$ (Figure 5 and Table 4). This model has 5 outliers (compared to 2 outliers in the Simple model), from which 4 out of the 5 appear as outliers also in the Detailed model 


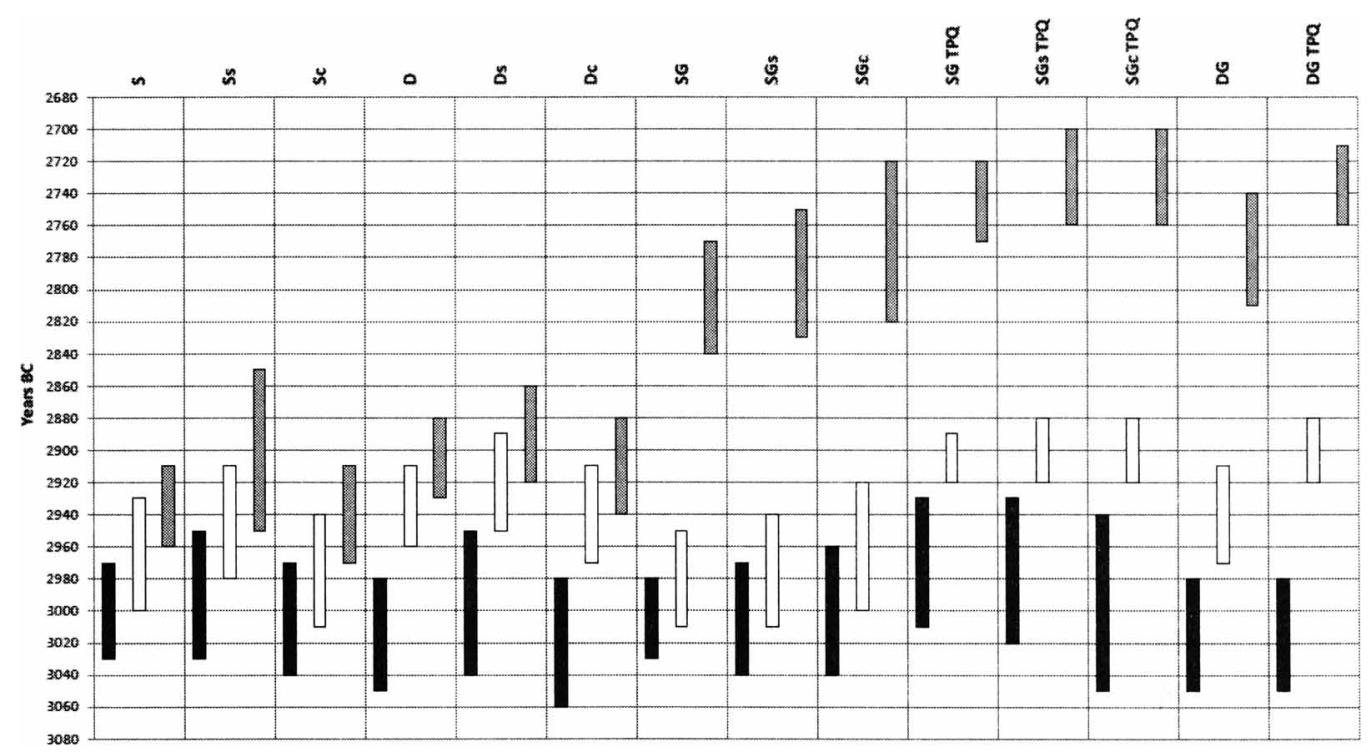

Figure 5 A summary of transition dates according to the various models. S: Simple model. s: Seeds samples only. c: Charcoal samples only. D: Detailed model. G: Gap included. TPQ: Terminus post quem. Black bars: EB I-II transition. White bars: End of EB II. Grey bars: Beginning of EB IIIB.

without the gap. Interestingly, the "Simple model of charcoal only with gap" yielded younger transitions than the "Simple model of seeds only with gap" model. This further supports that the oldwood effect at Tel Yarmuth is a minor one in general. When the same gap of $150 \pm 50 \mathrm{yr}$ was inserted in the Detailed model, the beginning of EB IIIB moved further downward to 2810-2740 BC, but retained nevertheless the 120-140 yr difference from its original model (Figure 6). Also in this case, the number of outliers increases from 5 to 7 outliers in the "Detailed model with gap."

The insertion of the $150 \pm 50 \mathrm{yr}$ gap into the various models resulted in a slight shortening of the EB II period, which is difficult to reconcile with current historical and archaeological reconstructions that ascribe for this period a much longer duration. In order to prevent an excessive shortening, it was decided to place a constraint for the end of the EB II period. Since the end of the EB II did not occur later than $2900 \pm 10 \mathrm{BC}$ in both the Simple and the Detailed models, it was decided to select this date as the most suitable terminus post quem (TPQ). As a result, in the Simple models with gap and TPQ, all periods shifted downwards by 20-70 yr. The beginning of the EB IIIB period shifts accordingly to $2770-2720 \mathrm{BC}$ in both the Simple and the Detailed models, irrespective of the type of sample, seeds or charcoal. Interestingly, the number of outliers in the Simple model with gap and TPQ is lower than in the Simple model with gap without TPQ (4 outliers instead of 5). On the other hand, in the Detailed model with gap and TPQ (Figure 7), 9 samples turn out to be outliers, compared to 7 outliers in the Detailed model with gap without TPQ.

\section{Transitions between Individual Archaeological Strata at Site According to Detailed Models}

When the Detailed models with seeds only or with charcoal only are run, the results for the boundaries are very similar. This implies that the old-wood effect is very small, if any. It is important to note that the sequence of archaeological phases in the 2 models is different. In the sequence of charcoal only, the Stratum B-2 is absent, while in the sequence of seeds only, the Stratum Bd-2 is absent (see Table 4). Therefore given the similarity of the boundaries between the 2 models, even with 


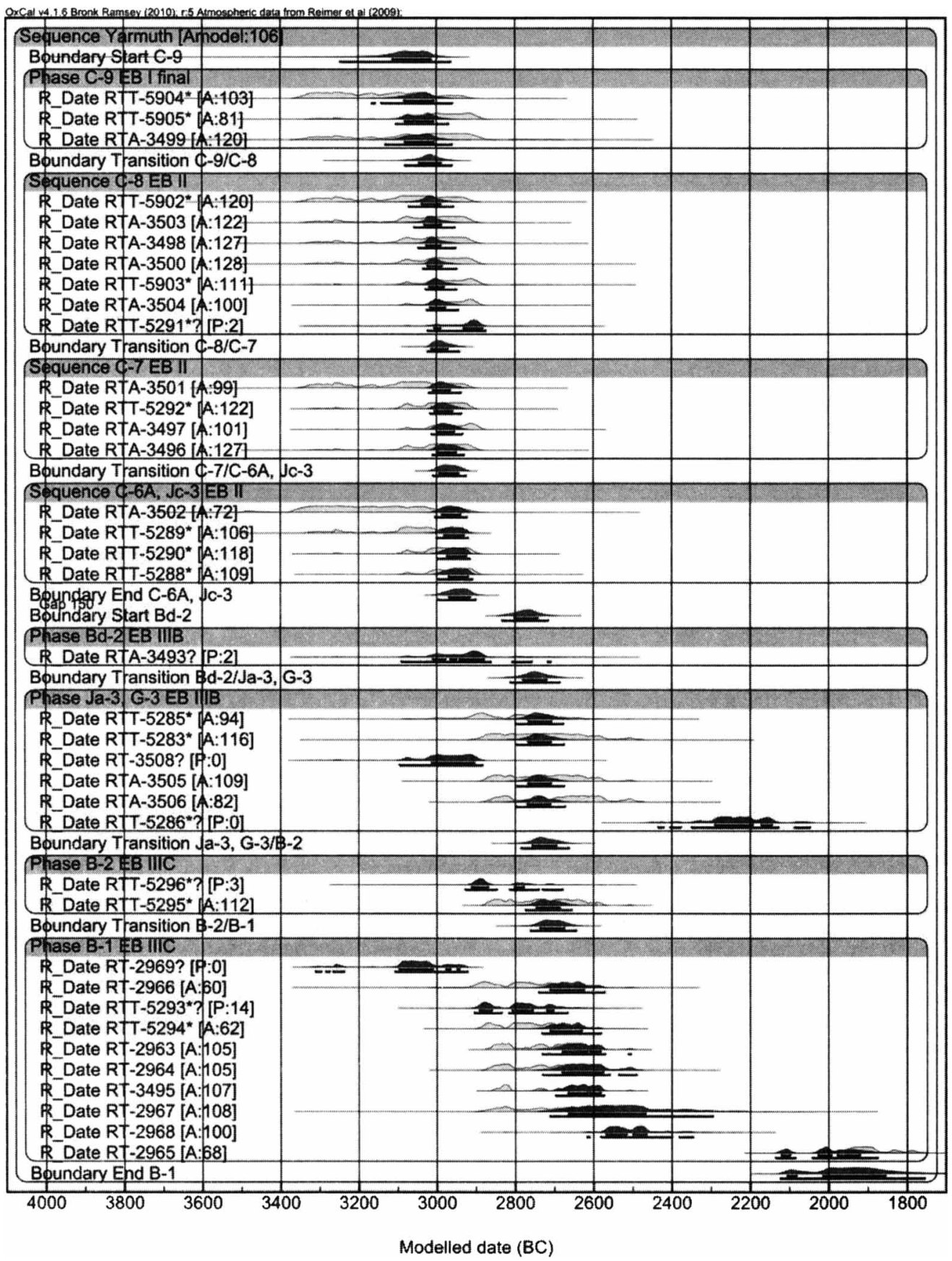

Figure 6 Detailed model with inserted gap of $150 \pm 50 \mathrm{yr}$

some differences in the strata, it was decided to build the final model using all the samples together, covering more phases. The models with gap and gap with TPQ were built using the same set of data. The ranges of the transitions as they appear in the various models are summarized in Table 4. 


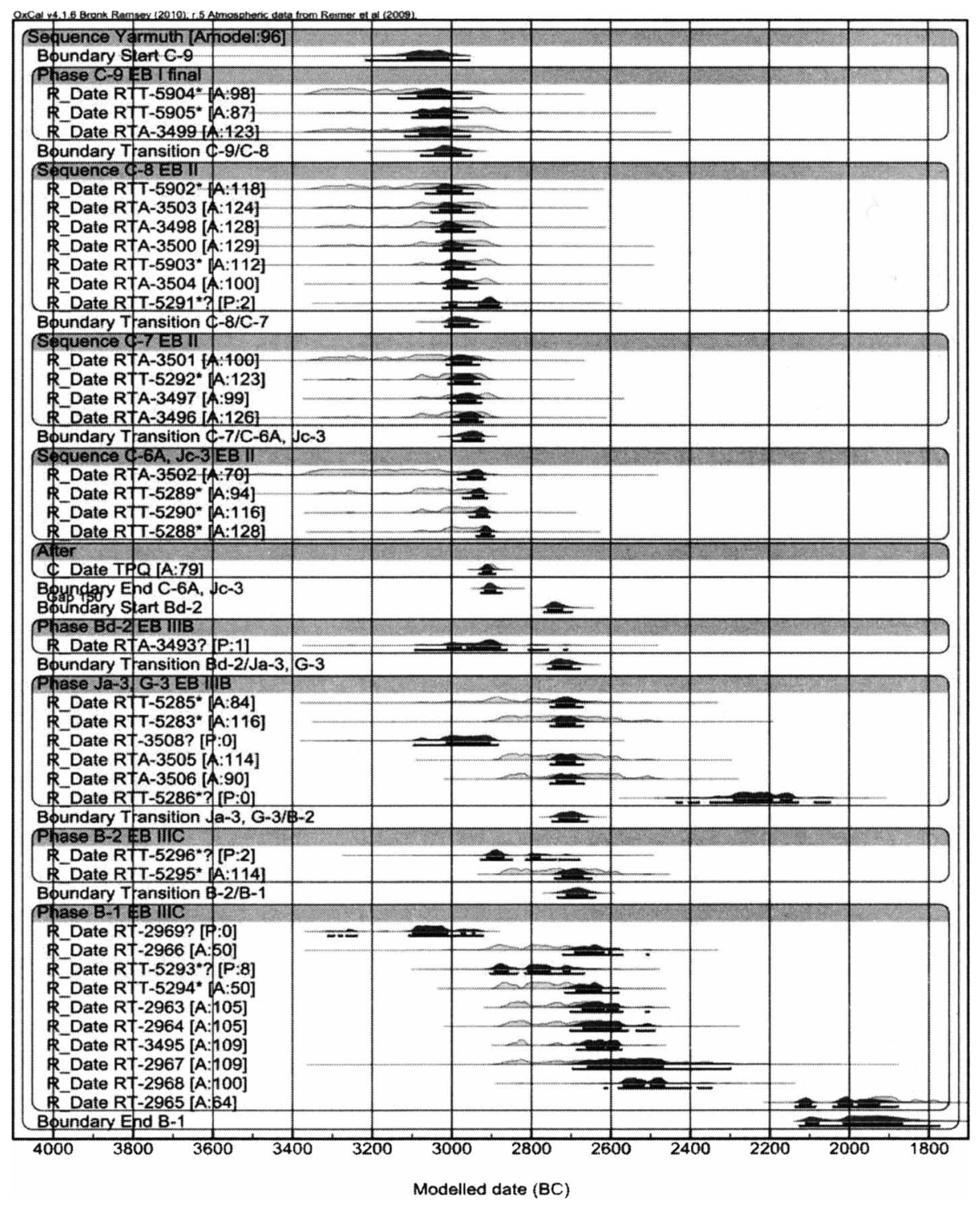

Figure 7 Detailed model with a gap of $150 \pm 50 \mathrm{yr}$ and a terminus post quem of $2900 \pm 10 \mathrm{BC}$

\section{DISCUSSION}

The 37 dates of Tel Yarmuth provide a good framework for the chronology of the Final EB I, the EB II, and the EB III periods in Israel. The Bayesian modeling applied to the Tel Yarmuth data set using the different approaches as explained above, identified from a minimum of 2 to a maximum of 9 outliers. By increasing the level of detail and constrains in the model, the number of outliers grows. The data indicate that at Tel Yarmuth the old-wood effect is not very significant, being between 20-60 yr in Simple models and $\sim 20 \mathrm{yr}$ in the Detailed models. The actual effect is probably due to a few specific samples that are clearly defined as outliers (especially RT-2969, RT-3508). 


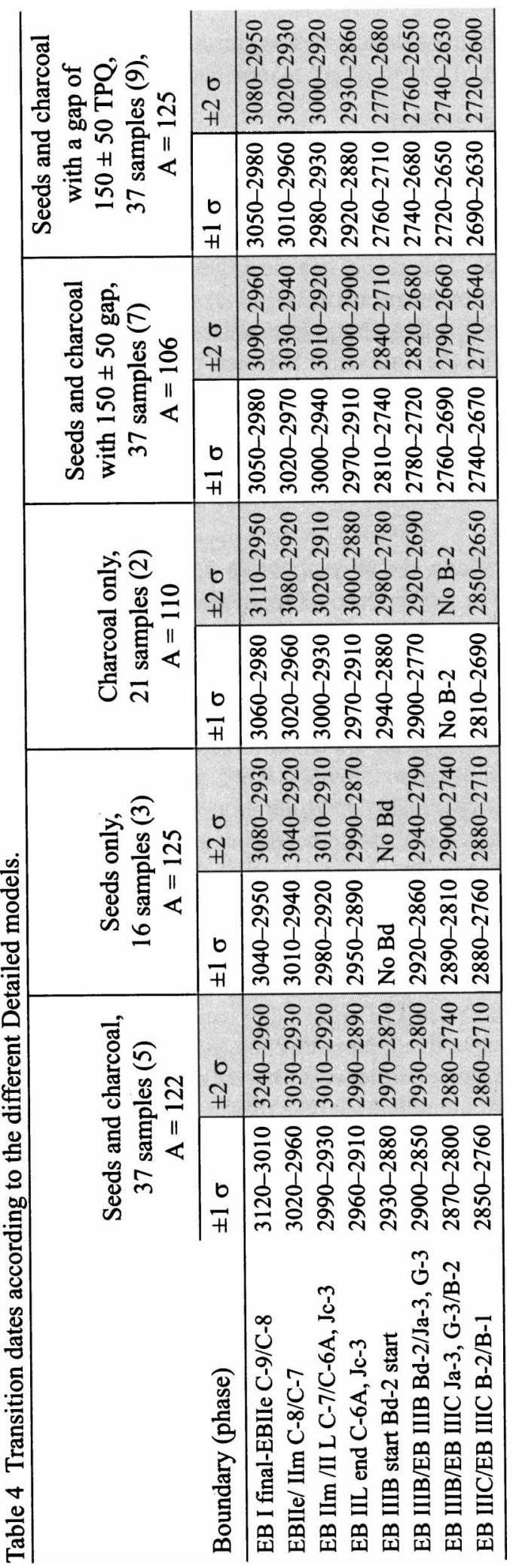


Based on all the different modeling performed, the models that best represent the archaeological stratigraphy and ${ }^{14} \mathrm{C}$ dates are

1. Detailed model (seeds and charcoal samples combined);

2. Detailed model with gap;

3. Detailed model with gap and TPQ.

The Detailed model, divided into 8 separate sequential layers, is built following the stratigraphy, and the boundaries are determined only by the ${ }^{14} \mathrm{C}$ dates. This model has the largest amount of data (32 samples) and a total of 5 outliers. In this model, the boundary between "Final EB I" and EB II falls around $3000 \mathrm{BC}$. The expected gap in the ${ }^{14} \mathrm{C}$ data (EB IIIA strata) is accounted for by the use of a sequential boundary between EB II and EB IIIB and the time range between the 2 boundaries is at most $170 \mathrm{yr}$ (maximum length individually for EB II is $140 \mathrm{yr}$ and for EB IIIA $80 \mathrm{yr}$; see Table 4), and most probably around 50-60 yr (EB II ends roughly 2930 BC and EB IIIB begins before $2880 \mathrm{BC}$ ). This is a completely different scenario for the EB II, which is traditionally given duration of about $300-400 \mathrm{yr}$, as well as the EB IIIA, allocated traditionally as 100-200 yr (e.g. de Miroschedji 2000:340).

Relying on historical and archaeological considerations, the 50-yr duration for EB IIIA is considered too short. The addition of a gap of $150 \pm 50 \mathrm{yr}$ in the Detailed model increased the EB IIIA duration to a maximum duration of $230 \mathrm{yr}$. But 2 more samples were turned into outliers (RTT-3493 charcoal, RTT-5293 short-lived), and the beginning of EB IIIB moved to between 2810-2740 BC.

In the Detailed model with gap, the end of EB II remains high and an attempt was made to stabilize it around $2900 \mathrm{BC}$ by inserting a TPQ for the beginning of EB IIIA. This created 2 additional outliers (total 9 outliers), but lowered the beginning of EB IIIB to 2760-2710 BC. These boundaries are closer to the conventional dates, and they leave a reasonably long duration for the EB IIIA and EB II. This identification of 2 more samples as outliers, with no contextual or archaeological explanation raises the question about the other contexts used in the model. Based on the comparison of the various models, and the raw ${ }^{14} \mathrm{C}$ data in this study, it is not straightforward to select the Detailed with gap and TPQ model instead of the Detailed model.

Among the $15{ }^{14} \mathrm{C}$ measurements (charcoal and seeds) made on samples from the EB II at Tel Yarmuth, none has a calibrated $1 \sigma$ range later than $2870 \mathrm{BC}$. On the other hand, the EB IIIB dates mostly do not start before 2900 BC (except 3 charcoal samples RT-2969, RT-3508, RTA-3493). Thus, the end of the EB II at Tel Yarmuth can be quite confidently established around $2900 \mathrm{BC}$, some $200 \mathrm{yr}$ earlier than conventionally thought. It is important to note that the end of EB II seems to have taken place precisely when a deep slope exists in the calibration curve, occurring between 2910-2890 BC (corresponding to $4350-4200$ uncalibrated). The fact that the measurements are neatly divided on the opposite sides of this slope further adds to the reliability of the date of the transition. The "EB IIIB begin" date can be slightly more flexible, but seems to have taken place in the course of the 29th century, or even during the 28th century. The latter date would imply that some of the ${ }^{14} \mathrm{C}$ samples are either not in situ or residual (RTT--5293, -5294) or there is a substantial oldwood effect (RTA-3493, RT-2966).

The end of the EB III in Tel Yarmuth is documented by a series of dates associated with the latest known pottery assemblage of EB IIIC as represented in Palace B1. The samples were gathered on plastered floors and are understood to form a uniform group of dates close to the time of abandonment of the site. All of the dates of this phase end before 2470 BC (except sample RT-2965 that is clearly intrusive). Actually most of the date ranges end before $2580 \mathrm{BC}$, and if the dates are really 
to be seen as an homogeneous group, the sample RT-2968 could be interpreted as secondary use of the ruins after the abandonment Tel Yarmuth. In this case, the end of EB IIIC could be even much earlier.

A lesson learnt from this study is that secure archaeological context is crucial for the accuracy of the model and reliability of the results. Detailed stratigraphy enables effectively to find out the archaeological outliers, like RTT-5286, which appears as an outlier only in the Detailed model. A case like this might bring attention to the context as open to intrusive samples.

The new chronological framework for the "Final EBI"- EB II, and EB III in Tel Yarmuth, taking in consideration the Detailed model and Detailed model with gap and TPQ, can be presented as below:

\begin{tabular}{ll}
\hline Transition "Final EB IB" to EB II: 3050 to 2980 BC \\
\hline End of EB II (= transition EB II-EB IIIA): 2950-2900 BC, most probably around 2900 BC \\
\hline Scenario 1 & Scenario 2 \\
Very short EB IIIA (max 70 yr) & EBIIIA of 150 yr duration \\
Beginning of EB IIIB: $2930-2880$ BC & Beginning of EB IIIB: 2760-2710 BC \\
End of EB IIIC: before mid-25th century BC & End of EB IIIC: before mid-25th century BC \\
\hline
\end{tabular}

\section{CONCLUSION}

Based on the modeling of the $37{ }^{14} \mathrm{C}$ dates from Tel Yarmuth, spanning the Early Bronze Age I, II, and III, a new chronology for this period has been proposed. The EB II has been shortened and its end is raised to about 2950-2900 BC. The EB III ends at the latest around $2450 \mathrm{BC}$, although according to the models an earlier date could be supported. The new ${ }^{14} \mathrm{C}$-based chronology of Tel Yarmuth, covering a major part of the EB period, represents a backbone for the EBA chronology in southern Levant.

\section{REFERENCES}

Aufderheide AC, Nissenbaum A, Cartmell L. 2004. Radiocarbon date recovery from bitumen-containing Egyptian embalming resins. Journal of the Society for the Study of Egyptian Antiquities 31:87-96.

Ben-Tor A. 1975. The first season of excavations at Tell Yarmuth: August 1970. Qedem (Monographs of the Institute of Archaeology, the Hebrew University of Jérusalem) 1:55-87.

Ben-Tor A. 1991. New light on the relations between Egypt and southern Palestine during the Early Bronze Age. Bulletin of the American Schools of Oriental Research 281:3-10.

Ben-Tor A. 1992. The Early Bronze Age. In: Ben-Tor A, editor. The Archaeology of Ancient Israel. New Haven: Yale University Press. p 81-125.

Bronk Ramsey C. 2009a. Bayesian analysis of radiocarbon dates. Radiocarbon 51(1):337-60.

Bronk Ramsey C. 2009b. Dealing with outliers and offsets in radiocarbon dating. Radiocarbon 51(3):102345.

Bronk Ramsey C, Dee MW, Rowland JM, Higham TFG, Harris SA, Brock F, Quiles A, Wild EM, Marcus ES,
Shortland AJ. 2010. Radiocarbon-based chronology for Dynastic Egypt. Science 328(5985):1554-7.

de Miroschedji P. 1999. Yarmuth, the dawn of city-states in southern Canaan. Near Eastern Archaeology 62(1): 2-19.

de Miroschedji P. 2000. An EB III pottery sequence for southern Israel. In: Philip G, Baird D, editors. Ceramics and Change in the Early Bronze Age of the Southern Levant. Sheffield: Sheffield Academic Press. p 315-45.

de Miroschedji P. 2003. The Late EB III Palace B1 at Tel Yarmuth: a descriptive summary. Eretz Israel 27:15370.

de Miroschedji P. 2008. Jarmuth Tel. In: Stern E, editor. The New Encyclopedia of Archaeological Excavations in the Holy Land, Volume 5, Supplementary Volume. Jerusalem: Israel Exploration Society. p 1792-7.

de Miroschedji P. In press a. Early Bronze Age (Israel, Palestinian territories). In: Killebrew A, Steiner M, editors. Oxford Handbook of Archaeology of the Levant (ca. 8000-332 BCE). Oxford: Oxford University Press. 
de Miroschedji P. In press b. Egypt and southern Canaan in the Third Millennium BCE: Uni's Asiatic campaigns revisited. In: Lehmann G, Gruber MI, Ahituv S, Talshir Z, editors. All the Wisdom of the East. Studies in Near Eastern Archaeology and History in Honor of Eliezer D. Oren. Orbis biblicus et orientalis XXX. Fribourg: Fribourg Academic Press.

de Miroschedji P, Davis S, Goldberg P, Kermorvant A, Nodet E, Rosen S, London G. 1988. Yarmouth 1, rapport sur les trois premières campagnes de fouilles à Tel Yarmouth (Israël), 1980-1982. Paris: Editions Recherche sur les civilisations.

Hennessy JB. 1967. The Foreign Relations of Palestine during the Early Bronze Age. Colt Archaeological Institute Publication. London: B. Quaritch.

Hornung E, Kraus R, Warburton DA. 2006. Ancient Egyptian Chronology. Leiden: Brill.

Jimenez Serrano A. 2007. Los Primeros Reyes y la Unifacacion de Egipto. Jaén: Universidad de Jaén.

Kitchen KA. 1991. The chronology of ancient Egypt. World Archaeology 23(2):201-8.

Mazar A. 1990. Archaeology of the Land of the Bible 10,000-586 B.C.E. New York: Doubleday.

Regev J, de Miroschedji P, Greenberg R, Braun E, Greenhut Z, Boaretto E. 2012. Chronology of the Early Bronze Age in the southern Levant: new analysis for a High Chronology. Radiocarbon, these proceedings.

Reimer PJ, Baillie MGL, Bard E, Bayliss A, Beck JW, Blackwell PG, Bronk Ramsey C, Buck CE, Burr GS, Edwards RL, Friedrich M, Grootes PM, Guilderson
TP, Hajdas I, Heaton TJ, Hogg AG, Hughen KA, Kaiser KF, Kromer B, McCormac FG, Manning SW, Reimer RW, Richards DA, Southon JR, Talamo S, Turney CSM, van der Plicht J, Weyhenmeyer CE. 2009. IntCal09 and Marine09 radiocarbon age calibration curves, 0-50,000 years cal BP. Radiocarbon 51(4): 1111-50.

Shaw I, editor. 2000. The Oxford History of Ancient Egypt. Oxford: Oxford University Press.

Sowada KN. 2009. Egypt in the Eastern Mediterranean during the Old Kingdom, An Archaeological Perspective. Orbis Biblicus et Orientalis 237). Fribourg: Fribourg Academic Press.

Stuiver M, Polach HA. 1977. Discussion: reporting of ${ }^{14} \mathrm{C}$ data. Radiocarbon 19(3):355-63.

van den Brink ECM, Levy TE. 2002. Interaction models, Egypt and the Canaanite periphery. In: van den Brink ECM, Levy TE, editors. Egypt and the Levant, Interrelations from the 4th through the Early 3rd Millennium BCE. london: Leicester University Press. p 338.

Wenke RJ. 2009. The Ancient Egyptian State, the Origins of Egyptian Culture (c.8000-2000 BC). Cambridge: Cambridge University Press.

Yizhaq M, Mintz G, Cohen I, Khalaily H, Weiner S, Boaretto E. 2005. Quality controlled radiocarbon dating of bones and charcoal from the early Pre-Pottery Neolithic B (PPNB) of Motza (Israel). Radiocarbon 47(2):193-206. 